\title{
A behavioral systems approach to adaptive computerized instructional design
}

\author{
ROGER D. RAY \\ Rollins College, Winter Park, Florida
}

\begin{abstract}
A computerized multimedia instructional system has been developed that adheres to behavioral systems principles for presenting both adaptive programmed instructional materials and laboratory simulations. This instructional software system, called MediaMatrix, is both an authoring environment and a presentation vehicle that adapts the complexity of presentations in real time to changes in a student's current rate of progress. It incorporates an automated knowledge-generation system that tracks all interactions between the user and any instructional objects within the system. Such knowledge is used both for a research database and for an artificial-intelligence engine that constructs an estimate of concept association networks (Verplanck, 1992a). Such networks reflect a learner's developing knowledge and skill base, and may be used for tutorial advising during student use.
\end{abstract}

Ray and Mitchell (1992) have previously described the goals and functions of an artificially intelligent, computerized system of multimedia teaching/learning resources that could be used in selective instructional and research environments. Their artificially intelligent multimedia resource library concept derives from a recognition that most teachers bring together a wide variety of resources, including a primary text, supplemental readings, lecture notes, media illustrations, and structured student activities, to help them in their teaching. Even laboratory exercises might be included as a means for helping students learn how to translate from declarative to procedural knowledge on a given topic.

By integrating such resources into a programmed instruction environment that also incorporates modern hypermedia computer technology and an artificial-intelligence engine to adapt to alternative learning rates and styles, this concept can be advanced even further. Ties to the emphasis of behavioral systems analysis on structural, functional, and operational principles (Ray \& Delprato, 1989) were detailed by Ray and Mitchell (1992), but details about software- or content-design implications of the systems approach with respect to programmed instructional applications were lacking. The present paper addresses these deficiencies both conceptually - by detailing how modern behavior principles may be used to guide adaptive programmed instructional content and presentation decisions - and proce-

The software authoring system MediaMatrix, described in this article, is owned and copyrighted by Learning Applications, Inc., and is available for preview at nominal cost, by contacting the author. The system is distributed as a shareware product that requires only a moderate charge for anyone using it for educational purposes within their own courses. A royalty-based licensing fee is required for commercial products, and is negotiated on a product-by-product basis by contacting the author. All requests should be addressed to R. D. Ray, Director, Institute for Learning Technology, Rollins College, Winter Park, FL 32789 (e-mail: rdray@rollins.edu). durally-by illustrating how the behavioral systems approach can guide development and applications of authoring software design.

\section{BEHAVIORAL SYSTEMS ANALYSIS}

Behavioral systems analysis has its conceptual and methodological roots in Kantor's interbehavioral philosophy, general systems theory, operant learning principles, and ethological assessment of behavioral patterning (Ray \& Delprato, 1989). The behavioral systems approach stresses three types of organizational dynamics to be discerned from a systems analysis - structural, functional, and operational. Structural analysis defines the fundamental elements of a behavioral system as well as the stochastic rules that maintain their organizational coherence. In behavioral terms, this typically means devising a behavioral taxonomy and identifying both the concurrent integration of components within time and the conditional, or kinematic, probabilities that define patterns of sequential activity across time (e.g., Gottman \& Roy, 1990). Functional analysis focuses on the outcomes served by networks of structurally defined behavioral patterns, as well as on the emergent patterning of multiple functional outcomes as they occur concurrently and sequentially in space and time. Operational analysis determines operating characteristics of the system by time series and other mathematical techniques that detect cyclic driving functions, perturbations, decay or damping functions, and many other regularities in the system's operations across time.

\section{APPLICATIONS TO INSTRUCTIONAL SYSTEMS DESIGN}

Especially relevant to devising an adaptive approach to programmed instruction is the incorporation of operant response shaping procedures. Shaping is accomplished by 
designing instructional presentations that incorporate both appropriate prompts in the initial stages of acquisition and appropriate shaping of the required verbal and skill response repertoires. Such dynamic processes require the construction of multiple "tracks" of programmed instruction reflecting alternative stages of successive approximation. Such approximations successively move the learner from highly dependent learning (i.e., from needing highdensity prompting, emitting first-approximation responses, and requiring high-density reinforcing feedback) to totally independent learning (i.e., to emitting nonprompted and sophisticated responses with lean schedules of feedback). The learner is constantly moved from one level of programmed instructional density to another, as his/her performance dictates. As performance improves, fewer and more difficult questions are provided as content density increases and programming feedback density goes from "rich" to "lean." Through the incorporation of such adaptive shaping procedures (Skinner, 1951, 1954), students are taught not only content but also how to learn as a general process skill.

Such progress is accomplished quite systematically. Once high levels of learning proficiency are reached, questioning and its associated feedback become purely random and relatively low density. In early stages, each programmed instructional question is linked to single paragraphs of related content. Upon reaching the highest levels of programming, students are given "pop" quiz questions (i.e., questions presented on a variable temporal schedule) on any topic they have studied previously, regardless of the topic they are currently studying. Should proficiency drop off momentarily, the system adapts to this lowered performance by dropping the student down one level of programming density to aid him or her in acquiring what might be more difficult material. Should the student continue to encounter difficulties, increasingly densely programmed instructional tracks are visited, until the student reaches a point of highly prompted learning at which he or she may be reshaped and begin to improve again. Of course, branching to alternative text is also incorporated to prevent perseveration on abstruse material.

\section{Language Systems and Knowledge Networks}

In instructional applications of behavioral systems analysis, the fundamental elements of the system are defined by interactions between the student and all software objects, especially those representing specific linguistic terms. These specific linguistic terms, which Skinner (1957) called tacts and intraverbals, and which Verplanck (1992b) has more recently referred to as notates and notants, become the structural and functional units for the analysis of stochastic associative networks. Associative probabilities relating each topical term with other explanatory or otherwise-associated terms become a measure of the system's overall organization, both within the system itself and within the emerging history of a student's interactions with instructional objects. Within the context of a learning history, linguistic terms and their associative probabilities represent emergent conceptual networks which define the concrete properties of an individual's knowledge of the instructional system's content at any given time. Such networks may be measured through a special form of testing, which Verplanck (1992a) calls the word associate test. In the word associate test, a single term or phrase is offered as a question to which the student is requested to supply a specified number of appropriately associated terms or graphical illustrations. Thus, for example, the name Pavlov might generate such associated terms as Russian physiologist, classical conditioning, salivary reflex, Nobel prize, and so forth.

Behavioral systems methodology also stresses the investigation of temporal operating characteristics in behavioral systems (Ray \& Delprato, 1989). This translates into investigations both of instructional object interaction velocity and of acquisition and fluency rates regarding questions posed by the system. Object interaction velocity will be discussed in more detail below. Fluency measurement is incorporated into the system directly as a shaping parameter by using different time-to-respond criteria in grading answers to programmed instructional questions, as well as criteria for determining whether a student should advance to a higher level of programmed instruction or not.

\section{Generative Training and Creativity}

In addition to incorporating a structured and intelligent adaptive learning system to shape independent study and improved reading comprehension skills, modern behavior theory instructs us as to how we might also teach creative problem solving. Epstein (1993) has identified four functional component skill categories that make up what he calls generative training. These categories, which derive from his extensive research on behavioral techniques for simulating traditionally defined cognitive skills, include capturing, challenging, broadening, and surrounding. Each of these can be used in guiding more effective instructional design - designs that build the foundations for emergent creative problem solving.

Capturing involves teaching the student to make small but important changes in the instructional system through interaction with it. At a low level, this may involve such activities as note taking and comment writing.

Challenging refers to the occasional use of surprise withholding of feedback or solutions, much as is designed into computer games. By trying ineffective behaviors in relatively "safe" circumstances (i.e., in an environment that does not belittle or ridicule the student for not succeeding), the learner finds new and more effective solutions through an almost automated self-shaping process (a process that the animal literature refers to as autoshaping). Thus, any shaping designed into computerized systems should also allow for some amount of exploratory self-shaping in selected areas. Students should find some degree of exploratory challenge available once they have gained the requisite foundational skills to avoid extinguishing on the system in the face of delayed or withheld feedback. This is one of many functions of appropriately designed laboratory simulations. 
Broadening refers to the construction of diverse repertoires that can, in fact, compete with one another until selfselection processes put the right combination together to solve a problem. Broadening argues that with respect to a given topic, several facets and orientations should be supported (e.g., alternative organizational strategies by timeline, contributors, topic, or an expert's organizational view of a given content corpus).

Finally, surrounding implies that we have a rich and complex stimulus environment to afford many new, but systematically developing, challenges to the learner. The environment must be simple enough not to intimidate, but complex enough to encourage hours of persistent exploration and use of diverse resources that converge to establish a richer repertoire on any given topic.

Such goals define general criteria for guiding and/or judging the appropriateness of content generation, programmed instructional content chunking, and question selection, as well as presentational graphics and interactive simulations. But these goals also challenge us in terms of better and more robust presentation software design. It is to this topic that we now turn.

\section{MediaMatrix: A Working Adaptive Learning System}

A working computerized instructional software system that meets the above behavioral systems design specifications is now available. This instructional software system, called MediaMatrix, is both an authoring environment and a delivery vehicle of many talents. These talents include its ability to function as (1) a straightforward presentation system for lectures or noninteractive browsing; (2) a prompting guide for helping students to recognize critical content details and organizational features; (3) a system for programmed instructional tutoring which intelligently adapts to changing learner needs; and (4) a sophisticated computerized test administration and assessment system. In addition, the system incorporates an automated knowledgegeneration system that tracks all interactions between the system and the user. This database is also used by an artificial-intelligence engine that builds an estimate of concept association networks (Verplanck, 1992a) used to reflect a learner's developing knowledge and skills. Through such concept networks, the system builds its own image of what the learner is learning as he or she interacts with the system. Such emergent concept networks can then be compared with reference, or "expert," networks both as a learning diagnostic and in order to generate further "advise" functions with respect to perceived gaps in the user's developing knowledge, wrongly developing concept associations, and unexplored concept-formation opportunities within the system.

A working instructional module on the topic of learning has been developed that demonstrates how both the executional design of MediaMatrix and the pedagogical- and content-design guidelines discussed above converge to make a powerful new computerized system of instruction.

All modules open with a request for the user to input a student identification number or name. This input ties all usage to the student's file folder, which resides inside the MediaMatrix folder that is, itself, maintained inside the system's preferences folder. Each student's folder maintains all historical data on that user, including a full history of interaction with every object encountered, as well as last mode of use. If the student is new, a new student folder, with all appropriate files and a default user mode, is created upon identification entry.

After inputting identification, the student is shown a frontispiece screen with titles, authors, and all appropriate pop-up buttons to establish a desired state of use. Students have several options, including mode of use, level of material, and means of navigation. With respect to mode of use, students can choose a very basic browse mode, or they can add a movie guide mode, enter the adaptive programmed instructional tutor mode, set the system to randomly ask questions related to topics previously studied via a probe mode, or take a predetermined quiz through assess mode.

In addition to mode selections, the student has the option of using any one of three levels of content detail - namely, a presentation level (a mere skeletal outline in bulleted presentation format), a notes level (that adds annotations and elaborations to the skeletal outline), and a text level (that presents a full text comparable to any textbook's section).

The content of the module is especially written in hypertext format, and includes integrative summaries that function as menus. This allows for alternative navigational strategies for reaching any given kernel of topical coverage. Thus, another pop-up button is always present that allows the student to access any of several alternative navigational maps, including a book's table of contents, a topics index, a timeline, a people index, and even a geographic map. This, of course, implies that every topical kernel has information with respect to: what (topic); who (persons contributing to topic); where (relevant geographical information, such as university, city, or country-e.g., the identification of Pavlov as a Russian working primarily at the Institute for Experimental Medicine in Leningrad); and when (e.g., it is noted that Pavlov won his Nobel prize in 1904); and why (i.e., the details and significance of the material being presented are discussed).

A more complete and comprehensive coverage of the topics typically included in an introduction to psychology course is in progress, and the MediaMatrix software system will be available (it is hoped by the time this article is published) to teachers and instructional-content authors for their own developmental activities, using either Microsoft Windows or Apple Macintosh platforms (see author note for details). In its presently available form, MediaMatrix requires developers to have HyperCard 2.2, a color Macintosh computer, a minimum of $8 \mathrm{Mb}$ RAM, and System 7 or higher. The Macintosh version uses HyperCard as its authoring environment, and the Windows version uses Asymetrix's Toolbook. The system is especially designed to create stand-alone applications that operate from CDROMs as the storage, usage, and distributional medium.

\section{Research Possibilities}

As noted earlier, MediaMatrix incorporates a knowledgegeneration engine capable of supporting the most sophis- 
ticated of behavioral systems research on user dynamics, content preferences, and design effectiveness. Even such operating characteristics as the rate, or velocity, of object interaction by each student user may be assessed for cyclicity, decay, or potential relations to accuracy. Incorporated video clips may be assessed independently for frequency and proportion of viewing in passive browse mode, as well as for content comprehension in programmed instructional (tutorial) mode. We are in the pilot testing phase of the learning module described above, and from this effort are preparing a set of dataanalysis protocols as a part of the developer's tools. This will allow preestablished statistical summaries to be quickly generated to address the most salient questions suggested by the behavioral systems perspective.

\section{REFERENCES}

EPSTEIN, R. (1993). Generative theory and education. Educational Technology, 33, 40-45.
GotTman, J. M., \& Roy, A. K. (1990). Sequential analysis: A guide for behavioral researchers. New York: Cambridge University Press.

RAY, R. D., \& DelpRATo, D. J. (1989). Behavioral systems analysis: Methodological strategies and tactics. Behavioral Science, 34, 81 127.

RAY, R. D., \& Mitchell, N. (1992). BehSys Anal: An artificially intelligent hypermedia resource library for descriptive behavioral research. Behavior Research Methods, Instruments, \& Computers, 24, 180-189.

SkINNER, B. F. (1951, December). How to teach animals. Scientific American, 185, 26-29.

SKINNER, B. F. (1954). The science of learning and the art of teaching. Harvard Educational Review, 24, 86-97.

SkINNER, B. F. (1957). Verbal behavior. New York: Appleton-CenturyCrofts.

VERPLANCK, W. S. (1992a). A brief introduction to the word associate test. Analysis of Verbal Behavior, 10, 97-123.

VERPLANCK, W. S. (1992b). Verbal concept "mediators" as simple operants. Analysis of Verbal Behavior, 10, 45-68.

(Manuscript received November 29, 1994; revision accepted for publication January 27, 1995.) 